\title{
Predictors of Long-Term Mortality in Patients Hospitalized in an Intensive Cardiac Care Unit
}

\author{
A Special Role of Anemia and Iron Status
}

\author{
Ewa Uscinska, ${ }^{1}$ MD, Bożena Sobkowicz, ${ }^{1} \mathrm{MD}$, Anna Lisowska, ${ }^{1} \mathrm{MD}$, \\ Robert SAwicki, ${ }^{1}$ MD, Milena Dabrowska, ${ }^{2}$ MD, Maciej Szmitkowski, ${ }^{3}$ MD, \\ Wlodzimierz J. Musial, ${ }^{1}$ MD, and Agnieszka M. TycinsKa, ${ }^{1}$ MD
}

\begin{abstract}
SUMMARY
Patients admitted to an intensive cardiac care unit (ICCU) are a heterogeneous population with a high mortality rate. The aim of our study was to investigate which clinical, biochemical, and echocardiographic parameters routinely assessed may affect long-term mortality in a non-selected ICCU population.

A total of 392 patients hospitalized between 2008-2011 (mean age, $70 \pm 13.8$ years, $43 \%$ women) were consecutively and prospectively assessed with the following admission diagnoses: 168 with acute coronary syndromes (ACS), 122 with acute decompensated heart failure (ADHF), and 102 with other acute cardiac disorders. Patients were treated according to the current European Society of Cardiology (ESC) guidelines.

During a mean $29.3( \pm 18.9)$ months of observation, $152(38.8 \%)$ patients died and $7.9 \%$ of the patients needed a red blood cell transfusion (RBC Tx). Patients who died were significantly older and had lower baseline levels of hemoglobin $(\mathrm{Hb})$, serum iron concentration (SIC), total iron binding capacity (TIBC), cholesterol, and left ventricular ejection fraction (LVEF), as well as lower eGFR values, and higher white blood cell (WBC) counts and C-reactive protein (CRP) levels $(P<0.05)$. Predictors of death in multivariate regression analysis were age, Hb, LVEF, WBC, and CRP. The most powerful factor was hospitalization for non-ACS. The risk of long-term mortality increased with decreasing levels of $\mathrm{Hb}$ $(P<0.001)$, SIC $(P=0.001)$, TIBC $(P=0.009)$, and the need for RBC Tx $(P<0.001)$, as well as the diagnosis of ADHF $(P<0.001)$ and the absence of ACS $(P=0.007)$.

In ICCU patients, age, $\mathrm{Hb}$, parameters of iron status, and LVEF are strong predictors of long-term mortality. Among the ICCU population, patients with ACS diagnosis have better survival. (Int Heart J 2016; 57: 67-72)
\end{abstract}

Key words: ICCU patients, Hemoglobin, Serum iron concentration, Late mortality, Risk factors

$\mathrm{P}$ atients admitted to an intensive cardiac care unit (ICCU) are a heterogeneous population with a common feature - an acute cardiovascular (CV) state requiring urgent lifesaving medical management. The main causes of admission are acute coronary syndromes (ACS), acute decompensated heart failure (ADHF), and other $\mathrm{CV}$ diseases, such as arrhythmias, conduction disturbances, pulmonary embolism (PE), cardiac tamponade, or septic shock (SS). ${ }^{1,2)}$ The estimated inhospital or 30-day mortality rate among patients hospitalized in an ICCU is reported in the range $3.3-9.1 \%^{1,2)}$ and increases up to $15.9 \%$ after 12 months. ${ }^{2)}$ It seems to be reasonable and helpful to explore simple parameters which could act as prognostic indicators in patients with critical cardiac life-threatening conditions related to high mortality rate circumstances.

Limited and conflicting data are available on the occurrence and prognostic value of anemia in ICCU patients. Moreover, it is unclear whether anemia is an independent predictor of long-term mortality or only an indicator of bad outcome. On the other hand, it is unclear whether iron metabolism disturbances independently of hemoglobin $(\mathrm{Hb})$ level influence long-term prognosis and whether there is a place for iron status and $\mathrm{Hb}$ concentration correction as a routine management in patients with critical cardiac diseases.

The aim of the study was to assess which clinical, biochemical, and echocardiographic parameters routinely evaluated in ICCU patients might act as indicators of poor outcome in critically ill cardiac patients. Moreover, we sought to investigate the prognostic role of anemia and impaired iron status in long-term observation in ICCU patients.

\section{MeTHODS}

Study population: A total of 392 patients (mean age, $70 \pm$

From the Departments of ${ }^{1}$ Cardiology, ${ }^{2}$ Hematological Diagnostics, and ${ }^{3}$ Biochemical Diagnostics, Medical University of Bialystok, Bialystok, Poland. This work is supported by Medical University in Bialystok, Poland, No 123-53714L.

Address for correspondence: Agnieszka Malgorzata Tycinska, MD, Department of Cardiology, Medical University of Bialystok, ul. Sklodowskiej-Curie 24a, 15-276 Bialystok, Poland. E-mail: agnieszka.tycinska@gmail.com

Received for publication June 22, 2015. Revised and accepted August 19, 2015.

Released in advance online on J-STAGE December 17, 2015.

All rights reserved by the International Heart Journal Association. 
13.8 years, $43 \%$ women) consecutively admitted to our ICCU between March 2008 and December 2011 were prospectively analysed. Among this ICCU population, 168 patients were admitted due to ACS (48\% had ST-elevation myocardial infarction (STEMI), 38\% non-ST elevation myocardial infarction (NSTEMI), and 14\% unstable angina), 122 due to ADHF, and 102 with other acute cardiac disorders such as PE and SS. Among patients with acute myocardial infarction (AMI) 3.6\% had 3rd Killip class (pulmonary edema) and 6\% had 4th Killip class (cardiogenic shock). Among the patients with ADHF, worsening of severe valvular disease was the reason decompensation was diagnosed in $30(16.4 \%)$. In a great majority of ADHF patients, the reason for hospitalization was clinical deterioration of previously diagnosed left ventricular dysfunction either of ischemic origin or due to cardiomyopathy. Patients were treated according to the current ESC guidelines. Vital signs and blood analysis were assessed at admission. A detailed description of the study population as well as the general methods, blood sampling, and definitions of anemia and iron deficiency (ID) were given elsewhere. ${ }^{3)}$ Admission glycemia, total cholesterol (TC), and low density protein (LDL) cholesterol were also included in the analysis.

The study design was compliant with the Helsinki Declaration of 1975 as revised in 1996 and it was approved by the local institutional committee on human research (Institutional Review Board - Local Bioethics Committee of Bialystok Medical University). Informed consent was obtained from all participants.

Primary endpoint: The composite endpoint of death from any cause was evaluated during long-term follow-up. We considered all-cause mortality as a more useful parameter than major adverse cardiovascular events (MACE), because in such a heterogeneous population the majority could experience MACE in the long-term observation. The researcher who was responsible for the collection of individual endpoints was blinded to the $\mathrm{Hb}$ level. Data concerning mortality were obtained from the local population registry run by a government office.

Statistical analysis: The results for continuous variables (number, mean, median, standard deviation (SD), minimum, maximum) are shown in the Table. The normality of the distributions of continuous variables were evaluated using the Kolomogorov-Smirnov normality test. Assessment of whether continuous parameters were different in the death and survival groups was conducted based on the Mann-Whitney $U$ test for two independent groups for continuous variables not presenting a normal distribution. The impact of various parameters on the incidence of death was examined using a univariate logistic regression test, while the influence of several factors was assessed using multivariate logistic regression. To assess the likelihood of death analysis, Cox proportional hazards models were calculated on the basis of per 1 year old/\%/g/dL. KaplanMeier analysis was performed to assess differences in mortality in particular patient subgroups. The analysis was performed using the statistical software package Statistica 6.0, EpilInfo v. 6.0, accepting $P<0.05$ as significant.

\section{RESUltS}

Mean baseline $\mathrm{Hb}$ level was $11.8 \mathrm{~g} / \mathrm{dL}( \pm 2.2)$, serum iron concentration (SIC) $45.0 \mu \mathrm{g} / \mathrm{dL}( \pm 39.2)$, total iron binding ca- pacity (TIBC) $267.9 \mu \mathrm{g} / \mathrm{dL}( \pm 74.9)$, and transferrin saturation (TSAT) $17.7 \%$ ( \pm 16.7 ). The table shows the baseline patient characteristics and the differences between the death and survivor groups. The prevalences of anemia and ID in the whole study population were $64 \%$ and $63 \%$, respectively. ${ }^{3)}$ The prevalence of anemia was comparable among females and males (67.5\% versus $62.2 \%, P=0.28$, respectively) and higher in the ADHF group as compared to ACS patients $(69.9 \%$ versus $59.4 \%, P=0.08$, respectively). ID and lower TIBC were more prevalent in females as compared to males $(39.3 \%$ versus $25.3 \%, P=0.02$ and $39.1 \%$ versus $15.2 \%, P<0.001$, respectively). Iron deficiency was more frequent in ACS as compared to ADHF patients $(69.9 \%$ versus $62.1 \%$, respectively, $P=$ $0.19)$. Thirty-one $(7.9 \%)$ patients needed red blood cells transfusion (RBC Tx); 8 survivors (2.0\%) and 23 patients who died (5.9\%). The indication for RBC Tx was a decrease in the $\mathrm{Hb}$ level below $7.0 \mathrm{~g} / \mathrm{dL}$ or hematocrit (Ht) level below $25 \%$.

During the mean $29.3( \pm 18.9)$ months of observation, $152(38.8 \%)$ patients died; $58(14.8 \%)$ in the ACS group and $88(22.4 \%)$ in the ADHF group. Patients who died were significantly older and had a longer mean time of hospitalization as compared to survivors (Table).

Patients who died had significantly lower levels of RBC, $\mathrm{Hb}, \mathrm{Ht}$, SIC, TIBC, TSAT, TC, LDL cholesterol, and left ventricular ejection fraction (LVEF), as well as higher creatinine and lower eGFR levels (Table), while survivors had significantly lower white blood cell (WBC) and C-reactive protein (CRP) levels (Table).

Logistic regression analysis: Among the clinical, biochemical, and echocardiographic parameters, multivariate regression analysis revealed that the highest risk of death for the whole study group was found for the changes in age, Hb, LVEF, WBC, and CRP (Figure 1). Moreover, a diagnosis other than ACS was related to an increased risk of death (Figure 1). In Cox regression analysis, age and LVEF were significant predictors of death: OR 1.021 (95\% CI: $1.003-1.04, P=0.022)$ and 0.98 (95\% CI: 0.96-0.99, $P=0.029)$, respectively.

Kaplan-Meier analysis: The risk of long-term mortality increased with decreasing levels of $\mathrm{Hb}(P<0.001)$, SIC $(P=$ $0.001)$, TIBC $(P=0.009)$, and the need for RBC Tx $(P<$ $0.001)$, as well as a diagnosis of $\operatorname{ADHF}(P<0.001)$ and the absence of $\operatorname{ACS}(P=0.007)$ (Figure 2).

\section{Discussion}

Our results show an overall high mortality rate in this ICCU population (particularly consisting of ACS and ADHF patients) after long-term observation lasting almost 2.5 years. One possible explanation for the poor clinical outcome in ICCU patients might be anemia, which is a frequent condition in patients suffering from critical illness. ${ }^{4)}$ To the best of our knowledge, this paper might be the first to have pointed out this important problem in a heterogeneous, difficult to treat ICCU population. The prevalence of anemia in patients with ACS varies from $17.5 \%$ to $28 \%$ at admission, ${ }^{5-7)}$ and usually increases during hospitalization. ${ }^{8,9)}$ In patients with ADHF, the incidence of low $\mathrm{Hb}$ level is even higher; $68 \%$ in men and $52 \%$ in women. ${ }^{10)}$ Although anemia is a known indicator of an unfavourable clinical profile related to inferior survival in both ACS and heart failure (HF) patients, ${ }^{5-7,11)}$ this correlation is complex. 
Table. Baseline Characteristics of the Study Patients - the Differences Between Deaths and Survivors

\begin{tabular}{|c|c|c|c|c|}
\hline $\begin{array}{l}\text { Parameter, } \\
\text { mean } \pm \mathrm{SD}\end{array}$ & $\begin{array}{l}\text { Whole study group } \\
\qquad n=392\end{array}$ & $\begin{array}{l}\text { Deaths } \\
n=152\end{array}$ & $\begin{array}{c}\text { Survivors } \\
n=240\end{array}$ & $P^{*}$ \\
\hline Time of hospitalization, days & $9.3( \pm 5.3)$ & $10.8( \pm 6.6)$ & $8.4( \pm 4.0)$ & $<0.001$ \\
\hline Age, years & $70.2( \pm 13.8)$ & $75.9( \pm 11.6)$ & $66.6( \pm 13.8)$ & $<0.001$ \\
\hline Clinical variables & & & & 0.097 \\
\hline $\mathrm{SBP}, \mathrm{mmHg}$ & $135.0( \pm 29.8)$ & $132.8( \pm 33.1)$ & $136.4( \pm 27.6)$ & \\
\hline $\mathrm{DBP}, \mathrm{mmHg}$ & $80.3( \pm 16.6)$ & $78.1( \pm 17.2)$ & $81.6( \pm 16.1)$ & 0.056 \\
\hline HR, bpm & $80.6( \pm 24.4)$ & $83.5( \pm 26.6)$ & $78.8( \pm 22.9)$ & 0.051 \\
\hline \multicolumn{5}{|l|}{ Biochemical variables } \\
\hline maximal troponin, ng/mL & $4.8( \pm 8.9)$ & $4.1( \pm 7.4)$ & $5.1( \pm 9.7)$ & 0.35 \\
\hline CRP, mg/L & $42.4( \pm 62.6)$ & $58.3( \pm 70.0)$ & $32.4( \pm 54.6)$ & $<0.001$ \\
\hline $\mathrm{BNP}, \mathrm{pg} / \mathrm{mL}$ & $707.0( \pm 1109.8)$ & $1394.0( \pm 1652.2)$ & $521.3( \pm 851.3)$ & 0.091 \\
\hline creatinine, $\mathrm{mg} / \mathrm{dL}$ & $1.2( \pm 0.7)$ & $1.4( \pm 0.8)$ & $1.1( \pm 0.6)$ & $<0.001$ \\
\hline eGFR, mL/minute $/ 1.73 \mathrm{~m}^{2}$ & $69.7( \pm 30.7)$ & $59.9( \pm 29.9)$ & $75.9( \pm 29.5)$ & $<0.001$ \\
\hline glucose, mg/dL & $101.1( \pm 62.4)$ & $107.1( \pm 68.2)$ & $97.4( \pm 58.3)$ & 0.07 \\
\hline $\mathrm{TC}, \mathrm{mg} / \mathrm{dL}$ & $129.3( \pm 73.3)$ & $109.2( \pm 74.0)$ & $141.9( \pm 70.0)$ & $<0.001$ \\
\hline $\mathrm{LDL}, \mathrm{mg} / \mathrm{dL}$ & $79.8( \pm 50.8)$ & $67.7( \pm 50.9)$ & $87.5( \pm 49.5)$ & $<0.001$ \\
\hline \multicolumn{5}{|l|}{ Peripheral morphology } \\
\hline $\mathrm{WBC}, \times 10^{3} / \mu \mathrm{L}$ & $10.0( \pm 4.3)$ & $11.1( \pm 5.1)$ & $9.4( \pm 3.6)$ & 0.003 \\
\hline $\mathrm{RBC}, \times 10^{6} / \mu \mathrm{L}$ & $4.0( \pm 0.7)$ & $3.9( \pm 0.7)$ & $4.1( \pm 0.7)$ & $<0.001$ \\
\hline $\mathrm{Hb}, \mathrm{g} / \mathrm{dL}$ & $11.8( \pm 2.2)$ & $11.2( \pm 2.1)$ & $12.1( \pm 2.2)$ & $<0.001$ \\
\hline $\mathrm{Ht}, \%$ & $36.0( \pm 6.4)$ & $34.4( \pm 6.2)$ & $37.0( \pm 6.3)$ & $<0.001$ \\
\hline $\mathrm{PLT}, \times 10^{3} / \mathrm{L}$ & $236.0( \pm 93.9)$ & $243.0( \pm 105.3)$ & $231.7( \pm 85.9)$ & 0.7 \\
\hline \multicolumn{5}{|l|}{ Parameters of iron status } \\
\hline $\mathrm{SIC}, \mu \mathrm{g} / \mathrm{dL}$ & $45.0( \pm 39.2)$ & $35.8( \pm 33.6)$ & $50.7( \pm 41.1)$ & $<0.001$ \\
\hline $\mathrm{TIBC}, \mu \mathrm{g} / \mathrm{dL}$ & $267.9( \pm 74.9)$ & $252.6( \pm 77.9)$ & $278.6( \pm 71.5)$ & 0.002 \\
\hline TSAT, $\%$ & $17.7( \pm 16.7)$ & $14.8( \pm 15.3)$ & $19.6( \pm 17.1)$ & $<0.001$ \\
\hline LVEF, \% & $42.3( \pm 14.9)$ & $38.7( \pm 15.5)$ & $44.5( \pm 14.0)$ & $<0.001$ \\
\hline
\end{tabular}

$P^{*}$ between deaths and survivors, Mann-Whitney test. BNP indicates B-type natriuretic peptide; CRP, C-reactive protein; DBP, diastolic blood pressure; eGFR, estimated glomerular filtration rate; Hb, hemoglobin; HR, heart rate; Ht, hematocrit; LDL, low density lipoprotein cholesterol; LVEF, left ventricular ejection fraction; PLT, platelet count; RBC, red blood cells; SBP, systolic blood pressure; SIC, serum iron concentration; SD, standard deviation; TC, total cholesterol; TIBC, total iron binding capacity; TSAT, transferrin saturation; and WBC, white blood cells.

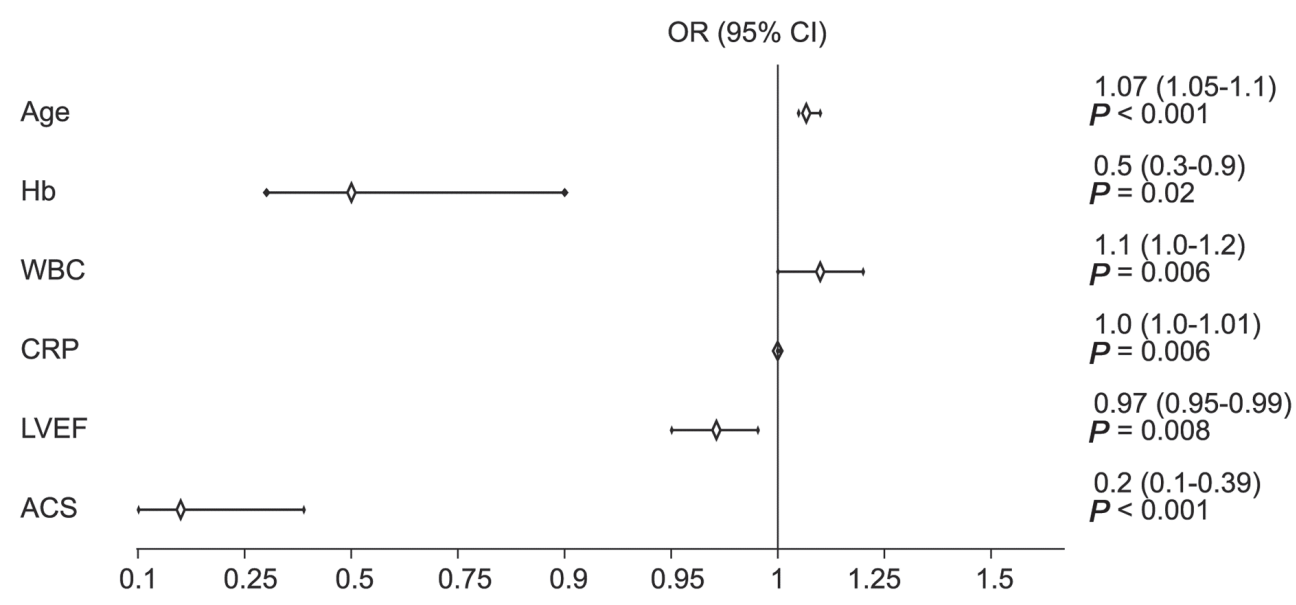

Figure 1. Multivariate regression analysis: independent variables influencing long-term mortality. Hb indicates hemoglobin; WBC, white blood cells; CRP, C-reactive protein; LVEF, left ventricular ejection fraction; ACS, acute coronary syndrome; OR, odds ratio; and CI, confidence interval.

There are multiple concomitant cardiac and extracardiac comorbidities, which may play a key role in the induction of mild-to-moderate anemia and increase the risk of death in these patients apart from $\mathrm{Hb}$ level. In our study, the prevalence of anemia was higher in the ADHF group as compared to the ACS group, however, the incidence of anemia in ACS patients was much higher than reported before. ${ }^{5-7)}$ Taking into consider- ation that hospital acquired anemia (HAA) has been reported in $18-57 \%$ of ACS patients ${ }^{8)}$ as well as anemia of inflammation, so called anemia of chronic disease (ACD), in HF patients could explain these results.

Iron metabolism disturbances are common in patients with cardiac disorders, ${ }^{12-15)}$ which was also confirmed in our previous study. ${ }^{3)}$ Recently, increasing attention has been fo- 

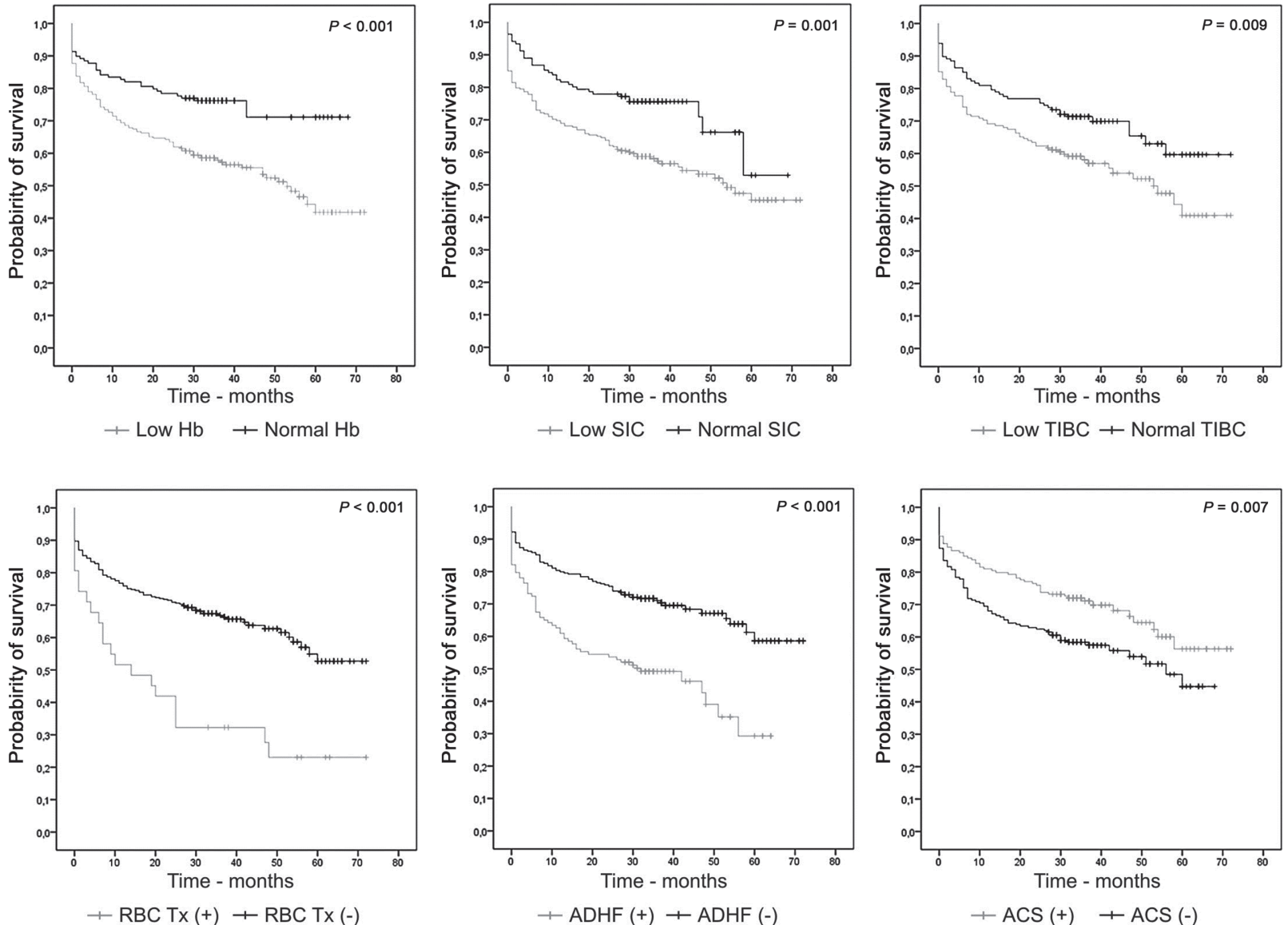

Figure 2. Kaplan-Meier survival curves: the differences in survival according to Hb, SIC, TIBC levels, and the need for RBC Tx, as well as the absence/ presence of ACS and ADHF. Hb indicates hemoglobin; SIC, serum iron concentration; TIBC, total iron binding capacity; RBC Tx, red blood cells transfusion; ACS, acute coronary syndrome; and ADHF, acute decompensated heart failure.

cused on iron deficiency anemia (IDA). ${ }^{12)}$ The studies have found a high incidence of ID in chronic heart failure (CHF) patients. ${ }^{16)}$ However, there are no clear data for the particular subgroups of ICCU patients. Iron deficiency in ICCU patients, as well as in those presenting with anemia, might be overlooked because assessing the parameters of iron metabolism is not always a routine practice. In our study, ID was more frequent in ACS patients than in ADHF patients. Thus, the potential cause of the higher incidence of anemia (but lower of ID) in the ADHF group could be the fact that the main cause of low $\mathrm{Hb}$ level might be chronic inflammation and many chronic comorbidities. On the other hand, in the ACS group the high prevalence of ID, but lower anemia, might be caused by blood loss due to invasive procedures as well as antithrombotic therapy. Patients who died, independently of basic clinical diagnosis, had lower parameters of morphology as well as iron metabolism, which was related to higher long-term mortality. A decrease in the $\mathrm{Hb}$ concentration of $1 \mathrm{~g} / \mathrm{dL}$ resulted in a $19 \%$ increase in mortality and a $1 \mu \mathrm{g} / \mathrm{dL}$ decrease in SIC worsened survival by $1 \%$. However, after adjustment of many variables routinely assessed at admission, anemia (more frequent in ADHF population), but not ID, was an independent risk factor for death. Patients who died had lower SIC and also lower TIBC (which is generally elevated in pure IDA, but usually de- creased in anemia of chronic disease - ACD). In the majority of ICCU patients with many chronic comorbidities, we deal with the compound cause of anemia (IDA/ACD) with a predominance of ACD, which can explain why low TIBC was an indicator of bad prognosis. ${ }^{3)}$

The importance of iron metabolism remains valid. Some data proved a favourable effect of intravenous iron treatment on exercise capacity, functional class, and LVEF, as well as renal function and quality of life, in either anemic or non-anemic patients presenting with $\mathrm{CHF}^{16-18)}$ Moreover, other studies have shown that iron replacement therapy improves prognosis. ${ }^{10,19)}$ Because anemia is highly prevalent in patients with acute cardiac states and is related to worse outcome, ICCU patients quite often require RBC transfusion (RBC Tx). ${ }^{20)}$ However, this may be related to adverse events and higher mortality. ${ }^{21)}$ Our results confirm this phenomenon. It is worth noting the recommendation of a restrictive strategy of RBC Tx with a cutoff in $\mathrm{Hb}$ level of $7 \mathrm{~g} / \mathrm{dL}$ in hemodynamically stable, nonacutely bleeding critically ill cardiac patients. ${ }^{22}$

Inflammation is involved in the initiation, progression, and instability of atherosclerotic plaques. ${ }^{23)} \mathrm{A}$ recent study has demonstrated that elevated serum hs-CRP levels at admission in patients with ACS were correlated with the severity and complexity of coronary atherosclerosis, LVEF, and troponin 
levels. ${ }^{24)}$ Several studies have suggested that in addition to aggressive statin therapy, CRP modulation might be one of the treatment options to reduce $\mathrm{CV}$ risk. There is no doubt that in AMI patients statin therapy significantly reduces the incidence of MACE, including cardiac death. There are also trials indicating, that among AMI patients with reduced LVEF, those with elevated levels of hs-CRP are more likely to benefit from statin treatment, while those with low levels of hs-CRP do not. ${ }^{25)}$ However, there are not enough data to recommend CRP reduction as a management target in at least HF patients. Surprisingly, our results showed that CRP as well as WBC were not the strongest predictors of long-term mortality in ICCU patients. The heterogeneity of the study population and a large proportion of patients with ADHF as well as other critical cardiac diseases might explain the lack of clinical significance of the inflammatory parameters in ICCU patients.

On the other hand, chronic kidney disease (CKD) is highly prevalent among patients with $\mathrm{HF}^{26)}$ Acute decompensation of HF may cause acute impairment of renal function. "Cardiorenal syndrome" is present in up to one-third of patients with $\mathrm{HF}$ and is related with prolonged hospitalization and worse survival. ${ }^{27)}$ There is evidence that baseline renal insufficiency rather than worsening of renal function was associated with an increased risk of death and death or rehospitalisation among patients with decompensated $\mathrm{HF}^{28)}$ The negative prognostic value of CKD was also proven in patients with acute myocardial infarction undergoing percutaneous coronary intervention (PCI); the presence of CKD was related to higher mortality and higher likelihood of multivessel coronary artery disease. ${ }^{29)}$ Moreover, in patients with CHF complicating AMI, renal dysfunction is one of the strongest discriminators of in-hospital mortality or occurrence of MACE one-month as well as oneyear after discharge. ${ }^{30)}$ Interestingly, our results show that parameters of renal function are not independent predictors of death in ICCU patients. However, it is worth noting that we analyzed only the admission values of renal function.

We also observed a trend towards increased glucose levels in patients who died, but it was not statistically significant. High admission glucose concentrations occur in patients with known or unrecognized diabetes mellitus, but also may be a result of elevated catecholamine and cortisol levels in acute life-threatening conditions. Other studies have reported the relations between admission glucose levels and long-term prognosis in diabetic patients with acute myocardial infarction ${ }^{31)}$ and also in a general high-risk cardiac population. ${ }^{2)}$ In our study, glycemia was assessed at admission and thus was not always fasting. This could be the reason why it was not an independent risk factor.

There is not enough data about the impact of lipid profile on survival in patients with acute cardiac diseases. However, low TC concentrations are strongly associated with increased mortality and morbidity among patients with ADHF. In our study, low TC as well as low LDL cholesterol were rather indicators of poor outcome, but not independent risk factors, which was also confirmed by other authors. ${ }^{32)}$

In our study, hospitalization due to ADHF was related to worse prognosis as compared to ACS, which is convergent with the earlier data ${ }^{2)}$ and might be related to well established, ESC-guided, mostly invasive treatment applied to ACS patients. Moreover, impaired systolic function, which defines HF with reduced ejection fraction (HF-rEF), correlates with a higher mortality rate. Our analysis showed that LVEF is still an important parameter in ICCU patients; even a slight decrease in LVEF resulted in a significant increase in the mortality rate and this correlation was independent of other variables in longtime observation. Surprisingly, in our study B-type natriuretic peptide (BNP), another parameter closely related to HF, was not an independent predictor of mortality. There is conflicting evidence about the predictive value of BNP/NT pro-BNP, but one of the most recent meta-analyses supports the hypothesis that natriuretic peptide-guided therapy can improve clinical outcomes in HF outpatients. ${ }^{33)}$

Conclusions: In ICCU patients, some of the variables routinely assessed at admission such as $\mathrm{Hb}$, parameters of iron status, and LVEF are strong predictors of long-term mortality. Other variables, like parameters of inflammation and renal function together with glycemia and cholesterol concentrations, are not clinically relevant. Among ICCU patients treated according to the current ESC guidelines, those with an ACS diagnosis have better survival.

\section{Disclosure}

Statement of competing interests: The authors declare that they have no conflicts of interest.

\section{REFERENCES}

1. Casella G, Cassin M, Chiarella F, et al; BLITZ-3 Investigators. Epidemiology and patterns of care of patients admitted to Italian Intensive Cardiac Care units: the BLITZ-3 registry. J Cardiovasc Med (Hagerstown) 2010; 11: 450-61.

2. Lipton JA, Barendse RJ, Van Domburg RT, et al. Hyperglycemia at admission and during hospital stay are independent risk factors for mortality in high risk cardiac patients admitted to an intensive cardiac care unit. Eur Heart J Acute Cardiovasc Care 2013; 2: 30613.

3. Uscinska E, Sobkowicz B, Sawicki R, et al. Parameters influencing in-hospital mortality in patients hospitalized in intensive cardiac care unit: is there an influence of anemia and iron deficiency? Intern Emerg Med 2015; 10: 337-44.

4. Anía BJ, Suman VJ, Fairbanks VF, Rademacher DM, Melton LJ 3rd. Incidence of anemia in older people: an epidemiologic study in a well-defined population. J Am Geriatr Soc 1997; 45: 825-31.

5. Uchida $Y$, Ichimiya $\mathrm{S}$, Ishii $\mathrm{H}$, et al. Impact of admission anemia on coronary microcirculation and clinical outcomes in patients with ST segment elevation myocardial infarction undergoing primary percutaneous coronary intervention. Int Heart J 2015; 56 : 381-8.

6. Anker SD, Voors A, Okonko D, et al; OPTIMAAL Investigators. Prevalence, incidence, and prognostic value of anaemia in patients after an acute myocardial infarction: data from the OPTIMAAL trial. Eur Heart J 2009; 30: 1331-9.

7. Lawler PR, Filion KB, Dourian T, Atallah R, Garfinkle M, Eisenberg MJ. Anemia and mortality in acute coronary syndromes: a systematic review and meta-analysis. Am Heart J 2013; 165: 14353. (Review)

8. Salisbury AC, Reid KJ, Amin AP, Spertus JA, Kosiborod M. Variation in the incidence of hospital-acquired anemia during hospitalization with acute myocardial infarction (data from 57 US hospitals). Am J Cardiol 2014; 113: 1130-6.

9. Salisbury AC, Amin AP, Reid KJ, et al. Hospital-acquired anemia and in-hospital mortality in patients with acute myocardial infarction. Am Heart J 2011; 162: 300-9. 
10. Cohen-Solal A, Leclercq C, Deray G, et al. Iron deficiency: an emerging therapeutic target in heart failure. Heart 2014; 100: 1414-20. (Review)

11. van der Meer P, Postmus D, Ponikowski P, et al. The predictive value of short-term changes in hemoglobin concentration in patients presenting with acute decompensated heart failure. J Am Coll Cardiol 2013; 61: 1973-81.

12. Andrès E, Federici L, Serraj K, Kaltenbach G. Update of nutrientdeficiency anemia in elderly patients. Eur J Intern Med 2008; 19: 488-93. (Review)

13. Weiss G, Goodnough LT. Anemia of chronic disease. N Engl J Med 2005; 352: 1011-23. (Review)

14. Weiss G. Iron metabolism in the anemia of chronic disease. Biochim Biophys Acta 2009; 1790: 682-93. (Review)

15. Arosio P, Levi S. Ferritin, iron homeostasis, and oxidative damage. Free Radic Biol Med 2002; 33: 457-63. (Review)

16. Cohen-Solal A, Damy T, Terbah M, et al. High prevalence of iron deficiency in patients with acute decompensated heart failure. Eur J Heart Fail 2014; 16: 984-91.

17. Filippatos G, Farmakis D, Colet JC, et al. Intravenous ferric carboxymaltose in iron-deficient chronic heart failure patients with and without anaemia: a subanalysis of the FAIR-HF trial. Eur J Heart Fail 2013; 15: 1267-76.

18. Anker SD, Comin Colet J, Filippatos G, et al; FAIR-HF Trial Investigators. Ferric carboxymaltose in patients with heart failure and iron deficiency. N Engl J Med 2009; 361: 2436-48.

19. Carrasco Sánchez FJ, Recio Iglesias J, Grau Amorós J. Complementary treatment of acute heart failure in patients with diabetes, chronic obstructive pulmonary disease or anemia. Med Clin (Barc) 2014; 142: 59-65. (Review)

20. Vincent JL, Sakr Y, Lelubre C. The future of observational research and randomized controlled trials in red blood cell transfusion medicine. Shock 2014; 41: 98-101.

21. Chatterjee S, Wetterslev J, Sharma A, Lichstein E, Mukherjee D. Association of blood transfusion with increased mortality in myocardial infarction: a meta-analysis and diversity-adjusted study sequential analysis. JAMA Intern Med 2013; 173: 132-9.

22. McIntyre L, Tinmouth AT, Fergusson DA. Blood component transfusion in critically ill patients. Curr Opin Crit Care 2013; 19: 326-33. (Review)

23. Ross R. Atherosclerosis--an inflammatory disease. N Engl J Med 1999; 340: 115-26. (Review)
24. Karadeniz M, Duran M, Akyel A, et al. High sensitive CRP level is associated with intermediate and high syntax score in patients with acute coronary syndrome. Int Heart J 2015; 56: 377-80.

25. Jeong HC, Ahn Y, Park KH, et al. Effect of statin treatment in patients with acute myocardial infarction and left ventricular systolic dysfunction according to the level of high-sensitivity C-reactive protein. Int Heart J 2014; 55: 106-12.

26. Matsue Y, Matsue Y, Matsumura A, et al. Prognostic implications of chronic kidney disease and anemia after percutaneous coronary intervention in acute myocardial infarction patients. Heart Vessels 2013; 28: 19-26.

27. Ronco C, McCullough P, Anker SD, et al; Acute Dialysis Quality Initiative (ADQI) consensus group. Cardio-renal syndromes: report from the consensus conference of the acute dialysis quality initiative. Eur Heart J 2010; 31: 703-11.

28. Nohria A, Hasselblad V, Stebbins A, et al. Cardiorenal interactions: insights from the ESCAPE trial. J Am Coll Cardiol 2008; 51: $1268-74$

29. Shiraishi J, Kohno Y, Nakamura T, et al. Prognostic impact of chronic kidney disease and anemia at admission on in-hospital outcomes after primary percutaneous coronary intervention for acute myocardial infarction. Int Heart J 2014; 55: 301-6.

30. Kim CS, Kim MJ, Kang YU, et al. Influence of renal dysfunction on clinical outcomes in patients with congestive heart failure complicating acute myocardial infarction. Int Heart J 2013; 54: 30410.

31. Malmberg K, Norhammar A, Wedel H, Rydén L. Glycometabolic state at admission: important risk marker of mortality in conventionally treated patients with diabetes mellitus and acute myocardial infarction: long-term results from the Diabetes and InsulinGlucose infusion in Acute Myocardial Infarction (DIGAMI) study. Circulation 1999; 99: 2626-32.

32. Yoon CH, Youn TJ, Ahn S, et al; Korean Heart Failure Registry. Low serum total cholesterol level is a surrogate marker, but not a risk factor, for poor outcome in patients hospitalized with acute heart failure: a report from the Korean Heart Failure Registry. J Card Fail 2012; 18: 194-201.

33. De Vecchis R, Esposito C, Di Biase G, Ariano C, Giasi A, Cioppa C. B-type natriuretic peptide-guided versus symptom-guided therapy in outpatients with chronic heart failure: a systematic review with meta-analysis. J Cardiovasc Med (Hagerstown) 2014; 15: 122-34. (Review) 